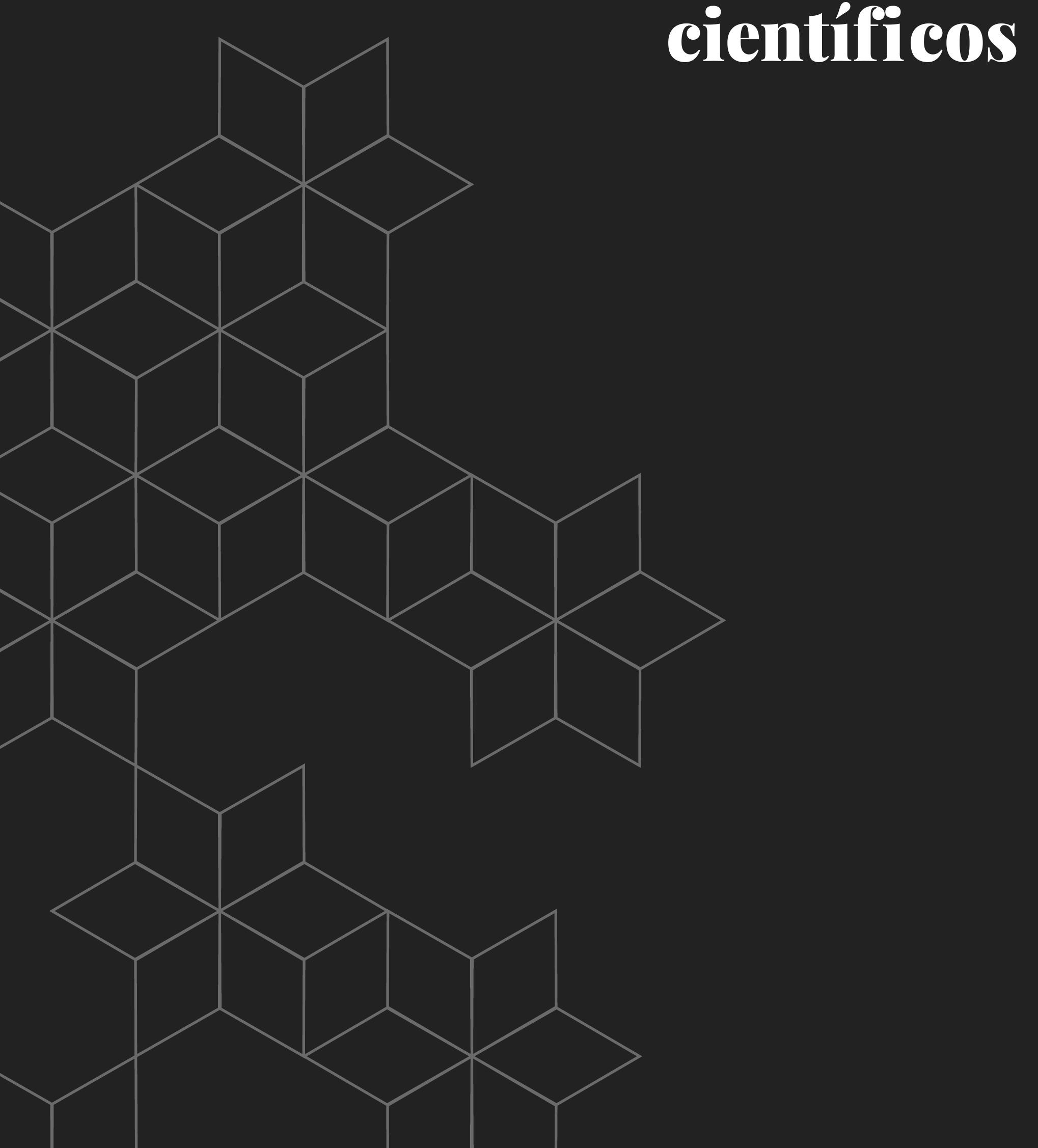




\section{Fijación transpedicular unilateral con fusión intersomática transformacional (Unilif): Serie prospectiva en 52 pacientes}

Dr. Mario Cahueque ${ }^{1}$

Dr. Enrique Azmitia ${ }^{2}$

Dr. Juan Armas 3

Dr. William López ${ }^{4}$

\section{Resumen}

El éxito de una fusión lumbar depende de la buena selección de pacientes. Instrumentación transpedicular bilateral con fusión con espaciador intersomático transforaminal, es ahora popular, debido a que mejora los índices de fisión, elimina la necesidad de dispositivos ortopédicos post operatorios y permite la movilización temprana. Sin embargo, el estrés causado por la fijación interna rígida se ha evidenciado que podría producir osteopenia y degeneración del segmento adyacente. Tiempos quirúrgicos prolongados, complicaciones transoperatorias y los costos de cirugía aumentan cuando se realiza fijación transpedicular. El siguiente reporte es un estudio prospectivo de 52 pacientes, con degeneración lumbar degenerativa, tratados con fijación pedicular unilateral con dispositivo intersomático (Unilif), en donde se pudo examinar los resultados, el índice de fusión y las complicaciones. La información radiológica y clínica se recolectó de forma prospectiva en pacientes con enfermedad lumbar degenerativa manejados con Unilif, desde enero 2014 hasta enero 2018. Preoperatoriamente y a los dos a los años, se registró la escala de Oswestri (ODI) y la Escala Visual Análoga (VAS). La adecuada fusión fue evidenciada y analizada por medio de radiografías y en algunos casos en tomografía. El tiempo promedio de cirugía fue de 93.4 minutos \pm 16.8 , la pérdida promedio de sangre fue de 130.8 $\mathrm{ml} \pm 210.9$, La escala de Oswestri y la Escala Visual Análoga mejoró significativamente $(p<0.005)$. El índice de fusión fue de $96.8 \%$ en análisis radiográfico y de $95.9 \%$ en análisis tomográficos. Un caso en donde el tornillo salió del pedículo fue reportado, sin embargo, el paciente no presentó dolor, fue tratado con reposo y con fisioterapia, con una evolución satisfactoria. Ninguna infección fue reportada. A pesar de ser un grupo pequeño, el seguimiento fue largo, la técnica

\footnotetext{
${ }^{1}$ Cirujano ortopedista y cirujano de columna, Spine Center, Guatemala.

2 Jefe del Departamento de Neurocirugía, Hospital Herrera Llerandi, Guatemala

${ }^{3}$ Médico residente y residente en investigación científica, Spine Center, Guatemala.

${ }^{4}$ Neurocirujano y cirujano de columna, Spine Center, Guatemala.
} 
Unilif ha probado ser efectiva y segura para los pacientes con enfermedad lumbar degenerativa.

Palabras clave: instrumentación pedicular unilateral, fusión intersomática, enfermedad lumbar, espondilolistesis, hernia de disco.

\section{Unilateral pedicle screw fixation with transforaminal interbody fusion (UNILIF): A 52-patient prospective series}

\section{Abstract}

A successful lumbar spine fusion depends on a good selection of patients. Bilateral pedicle instrumentation with transforaminal interbody fusion is now popular because it improves fusion rates, eliminates the need of post-operative orthopedic devices and allows early mobilization. However, it has been evidenced that stress caused by rigid internal fixation could lead to osteopenia and degeneration of adjacent segments. Long surgical procedures, postoperative complications and costs all increase when pedicle screw fixations are performed. The following report is a prospective study of 52 patients with lumbar degenerative disease who underwent unilateral pedicle fixation with the interbody device (Unilif). The study examines outcomes, fusion rates and complications. Clinical and radiological data was prospectively collected from January 2014 to January 2018 from patients with lumbar degenerative disease who were treated with Unilif. Both postoperatively and two years later, the study recorded the Oswestri Disability Index (ODI) and the Visual Analogue Scale (VAS). Proper fusion was determined and analyzed by means of radiography and in some cases of CT scans. Average surgery time was 93.4 minutes \pm 16.8 , average blood loss was $130.8 \mathrm{ml} \pm 210.9$, and the Oswestri Disability Index (ODI) and Visual Analogue Scale improved significantly $(p<0.005)$. The fusion rate was $96.8 \%$ in radiographic analyses and $95.9 \%$ in CT scan analyses. One single case was reported in which the screw became loose, however, the patient had no pain, was treated with rest and physiotherapy and had a satisfactory evolution. No infections were reported. Even though it was a small group with a long follow-up, the Unilif technique has proven to be effective and safe for patients with lumbar generative disease.

Key words: unilateral pedicle instrumentation, interbody fusion, lumbar disease spondylolysthesis, disc herniation. 


\section{Introducción}

Fusión transforaminal con dispositivo intersomático (TLIF) fue inicialmente descrita por Harms y Rolinger en 1982 (1). Ha resultado ser una de las técnicas estándar ordinarias para descompresión del foramen ipsilateral y para una apropiada fusión intersomática. Esta técnica permite una ventaja anatómica al no requerir una retracción grande del saco dural y de sus contenidos. Índices altos de fusión han sido reportados. Este procedimiento es normalmente acompañado por la colocación de tornillos transpediculares bilaterales en el segmento correspondiente; esto resulta en un segmento completamente rígido y la estabilización durará hasta que el segmento se fusione (2), (3). Algunos autores han probado que la rigidez excesiva, de tal fijación, puede poner en peligro el proceso de fusión debido a la resorción del injerto, esto se debe a la falta de tracción en la lámina del cuerpo vertebral (4), (5), (6).

La evidencia científica en la literatura ha establecido que la fijación del tornillo transpedicular unilateral, justo después de la fusión, produce resultados radiológicos favorables inmediatos. Si se compara con la fijación bilateral, esto se hace a un costo menor porque se usa menos cantidad de implantes (7), (8), (9), (10), (11). La Fusión Transforaminal Intersomática Lumbar Mínimamente Invasivo (MI-TLIF) fue descrita originalmente por Foley (12).

Es por eso que la fijación MISTLIF es requisito absoluto para el pedículo contralateral y, por lo tanto, causa a su controvertida. La Fijación Transpedicular Unilateral como complemento de un MISTLIF (técnica llamada Unilif) se ha descrito desde aproximadamente el año 2006 (13).

Ventajas teóricas de este enfoque unilateral posterior incluye: una disminución de pérdida de sangre, procedimiento quirúrgico más rápido, dosis de radiación reducida y, por supuesto, la preservación de la articulación posterior contralateral proceso contralateral. También tiene tasas de fusión radiográfica similares a las de TLIF abierto y una predisposición a producir mejores resultados clínicos en el inmediato postoperatorio (7), (8), (11), (14), (15). Para poder evaluar la clínica, con alta efectividad de la fijación unilateral del tornillo transpedicular más Fusión Transforaminal Intersomática Lumbar (Unilif) se tomó en el estudio a los pacientes con enfermedad lumbar degenerativa; el promedio del índice de discapacidad en la escala de Oswestry (ODI) y la evaluación del dolor lumbar, por medio de la Escala Visual Análoga (VAS), fue a los 2 años.

\section{Métodos}

De enero de 2014 a enero de 2018, se recolectó, de forma prospectiva, datos clínicos y radiológicos de pacientes con enfermedad lumbar degenerativa. La cual fue tratada por medio de Unilif de un nivel, en una institución privada, realizada por dos cirujanos de columna. Cada paciente firmó un formulario de consentimiento informado para poder participar en el estudio. Se realizó un estudio prospectivo de series de casos en 52 pacientes con enfermedad lumbar degenerativa. Dentro de los criterios de inclusión, se tomaron únicamente a pacientes mayores de 18 años y tratados por una artrodesis lumbar de un nivel para enfermedad lumbar degenerativa (pacientes con espondilolistesis de bajo grado, hernia discal recurrente, hernia de disco extraforaminal y estenosis lumbar con radiculopatía unilateral). Pacientes con estenosis central mayor o radiculopatía bilateral, con instrumentación previa de columna, tumores, dolor tipo radicular bilateral, obesidad (IMC> 30) y osteoporosis fueron exentos de este presente estudio. Es importante destacar que los pacientes con síntomas unilaterales de tipo radicular, con evidencia radiográfica de hernia foraminal bilateral, se incluyeron en este estudio, como pacientes con estenosis foraminal, en donde únicamente se incluyó el lado que presentaba sintomatología, el cual posteriormente, fue descomprimido de forma directa. En el presente estudio, el cien por ciento del seguimiento se llevó a cabo. Antes de la operación y a los 3 años, 
documentamos ODI y VAS lumbar y toda aquella escala visual análoga en pacientes con dolor de tipo radicular en las piernas. La fusión intersomática se analizó por medio de radiografía y una tomografía. La técnica quirúrgica fue la siguiente: con el paciente en decúbito prono, (boca abajo), bajo anestesia general y uso transoperatorio de imágenes de fluoroscopía intensificada con brazo $\mathrm{C}$ se realizó un abordaje quirúrgico, del lado sintomático. La incisión en piel fue como promedio de aproximadamente $3 \mathrm{~cm}$, se utilizó separadores de microdiscectomía. Se dio inicio colocando tornillos transpediculares del segmento afectado y un separador TLIF. Posteriormente, se realizó una osteotomía de la lámina y la faceta articular, se completó una facetectomía medial. Se localizó el triángulo de Kambi, la raíz medial y lateral se retrae por medio de separadores de raíz, se hizo la preparación y el injerto óseo se colocó en el espacio lumbar intersomático. Se implantó un dispositivo intersomático rectangular $\mathrm{o}$, en forma de banana, para finalmente concluir con la fusión unilateral, por medio de la colocación de tornillos transpediculares.

\section{Resultados}

Los resultados clínicos se evaluaron utilizando ODI y la Escala Visual Análoga (VAS) para el dolor radicular de piernas y espalda, los pacientes completaron un mínimo 2 años de seguimiento. ODI y VAS se analizaron a los $3,6,12,24$ y 36 meses, en el análisis estadístico se confirmó la normalidad de las variables. Las variables paramétricas se expresaron como media (desviación estándar), no paramétrica como mediana (rango intercuartil).

Las variables categóricas se llamaron absolutas y las frecuencias utilizadas fueron las frecuencias relativas. Se realizó una evaluación comparativa antes y después, a través de la prueba T-student. Los resultados se consideraron significativos en un valor de $p<0,05$. Los análisis se completaron al utilizar el programa estadístico SPSS software, versión 20 (SPSS Inc.).

Las características principales, se presentan en la tabla 1. La edad promedio de los pacientes, fue de 51.1 años, (29-60 años). La distribución por género fue 15 pacientes femeninos (26\%) y 37 pacientes masculinos (74\%). El promedio del Índice de Masa Corporal (IMC) fue 28. El lado más frecuente de la radiculopatía fue izquierdo. El segmento operado con mayor frecuencia fue L4 / L5 (85 \%), L5 / S1 (12 \%), L3 / L4 (3\%). Las principales indicaciones quirúrgicas fueron espondilolistesis de bajo grado, con radiculopatía unilateral lo cual representó 34 pacientes y en 7 pacientes se observó discopatía estenosis foraminal. El tiempo promedio de cirugía fue de $93.5 \mathrm{~min} \pm 16.8$, la pérdida de sangre promedio fue de $130.8 \mathrm{ml} \pm 210.9$.

Tabla 1

Datos demográficos

\begin{tabular}{ll} 
& Pacientes \\
Género & \\
\hline Mujer & $15(26 \%)$ \\
Hombre & $37(74 \%)$ \\
Edad promedio & $51.1(29-60)$ \\
IMC promedio & 28 \\
\hline Indicación & \\
\hline Espondilolisis de bajo grado & $34(65 \%)$ \\
Discopatía estenosis foraminal & $7(13 \%)$ \\
Hernia discal foraminal & $6(11 \%)$ \\
Recurrencia de hernia discal & $5(9 \%)$ \\
\hline Nivel de artrodesis & \\
\hline L3 / L4 & $2(3 \%)$ \\
L4 / L5 & $44(85 \%)$ \\
L5 / S1 & $6(12 \%)$
\end{tabular}


ODI y VAS mejoraron significativamente $(p<0.005)$ al final del seguimiento. En los primeros 3 meses, se evidenció un progreso significativo, que aumentó en los meses siguientes (tabla 2). El dolor radicular de piernas VAS mejoró en todos(as) los y las pacientes. Además de dolor lumbar ( $p<0,0001)$. El tiempo hospitalario promedio fue de 3 días, sin tener en cuenta el día de la cirugía. Pocas fueron las complicaciones, no se evidenció infecciones o fuga de líquido cefalorraquídeo. Las y los pacientes, al día siguiente de la cirugía, caminaron e inmediatamente y también se inició de forma casi inmediata, a las 24 horas, como parte de la movilización temprana, fisioterapia y rehabilitación. El drenaje de tipo hemovac se colocó rutinariamente. No hubo necesidad de realizar transfusiones sanguíneas a ninguno(a) de las y los pacientes. A los 3 años de seguimiento, la tasa de fusión fue del 95,1\% en análisis radiográfico y fue del 93,0 \% en el análisis de tomografía. Un aflojamiento de tornillo fue reportado, sin embargo, paciente asintomático y sin dolor, se trató con descanso y fisioterapia, al final del manejo conservador se pudo evidenciar buena evolución. No se mostró ninguna complicación por infecciones. Hubo una disminución considerable en el uso de analgésicos en los primeros 3 meses.

Tabla 2

Datos VAS

\begin{tabular}{cccc} 
Meses & $\begin{array}{c}\text { ODI } \\
\text { \#(pacientes) }\end{array}$ & VAS & VAS-dolor de pierna \\
Preoperatorio & $52.4(52)$ & 6.4 & 7.6 \\
\hline 6 & $23.5(52)$ & 3.8 & 1.7 \\
12 & $18.7(52)$ & 2.1 & 1.1 \\
\hline 24 & $18.4(36)$ & 1.9 & 1 \\
\hline 36 & $15,1(21)$ & 1,2 & 1 \\
\hline & $\mathrm{P}<0.05$ & $\mathrm{P}<0.05$ & $\mathrm{P}<0.05$ \\
\hline
\end{tabular}

\section{Discusión}

TLIF se describe como una alternativa quirúrgica regular para pacientes con espondilolistesis ístmica y dolor axial. El tornillo de fijación debiese de ir orientado, a lo largo con este procedimiento para brindar una mejor estabilidad. El tornillo de fijación bilateral se utilizó inicialmente, pero más investigaciones revelaron que la fijación unilateral, representa una buena opción para preservar la estabilidad de la columna vertebral (14), (16), (17). En la cirugía de fusión espinal, la necesidad de instrumentación unilateral o de la instrumentación bilateral es un tema controvertido. Estudios biomecánicos demostraron que la fijación unilateral, después de la fijación con TLIF, representa menos estabilidad rotacional y rigidez que el tornillo pedicular bilateral (6), (7), (17). Sin embargo, es frecuente la destrucción de tejido y estructuras cercanas, que exacerbaron considerablemente la inestabilidad de la columna vertebral, esto fue utilizado 
en estudios biomecánicos in vitro. Adicionalmente, el mantenimiento de la estabilidad lumbar simplemente dependía de la fijación transpedicular con tornillo unilateral, sin ningún dispositivo de soporte. En la actualidad, se ha llevado a cabo un consenso general, en el que la instrumentación unilateral debería limitarse a una fusión de un solo nivel y no extender la fusión multinivel debido a su fuerza inadecuada de fijación.

Kabins et al. (8) describe una fusión clínica y radiográfica similar, al comparar resultados entre instrumentación unilateral en comparación con el presente estudio Suk et al. (9) diseñó un estudio prospectivo con 87 pacientes para asociar la fijación bilateral con fijación unilateral de tornillo pedicular en uno o dos segmentos fusionados, la conclusión final fue, que la fijación unilateral es tan efectiva, como fijación de tornillo bilateral en todos los elementos evaluados: pérdida de sangre, funcionamiento y estabilidad de columna, tiempo quirúrgico, duración de la estancia hospitalaria, resultados clínicos, tasas de complicaciones y gastos médicos. Recientemente, Xue et al. (12) comparó clínica y resultados radiográficos, en pacientes con fijación transpedicular unilateral y bilateral, concluyó que TLIF con fijación unilateral de tornillo transpedicular, es una opción de tratamiento ventajosa con mejores resultados en términos de tiempo operatorio, pérdida de sangre y hospitalización por enfermedad de un solo nivel, informaron resultados coincidentes. Está claro que en los modelos in vitro, la rigidez obtenida por una fijación bilateral

22 es superior que unilateral.
Por otro lado, se demostró que la ausencia de cierto grado de movimiento puede dar lugar a una falla de fusión. En el seguimiento de 2 años, nuestra tasa de fusión intersomática, medida con los resultados obtenidos a través de Tomografía Computarizada, CT (93.0) son muy similares a los reportados por Lin et al. (92.3 \%) (18) o en el metanálisis de Xiao et al. de 270 casos (92.2 \%) (17). En pacientes, en los que aún no se observó una fusión completa, se mostraban clínicamente asintomáticos, por lo que no fue necesario una cirugía de revisión.

\section{Limitaciones}

El estudio actual tiene varias limitaciones, la más significativa es la falta de un grupo de control, el seguimiento es largo y se realizó de forma detallada y minuciosa por el mismo equipo. La población es pequeña y muy heterogénea, esto descarta pacientes obesos y pacientes de edad avanzada, quienes realizamos este estudio creemos que en este tipo de pacientes se debe considerar la instrumentación bilateral. 


\section{Conclusión}

Este estudio demuestra los beneficios de Unilif de un nivel es mínimamente invasivo en el tratamiento de los pacientes con enfermedad lumbar, sin estabilidad marcada de columna. Los resultados demostraron una baja tasa de morbilidad perioperatoria que, a su vez, se asocia con una recuperación acelerada. Los resultados clínicos y radiológicos fueron positivamente comparables. En el resultado a los 2 años, Unilif puede ser una valiosa alternativa quirúrgica para técnica de fijación de tornillos bipediculares en el manejo de enfermedades degenerativas lumbares, estables. Se requerirán revisiones adicionales para respaldar estos resultados.

\section{Conflictos de interés}

Los autores declaran que no tienen conflicto de intereses.

\section{Referencias}

1. Harms $\mathrm{J}$, Rolinger $\mathrm{H}$. A one-stager procedure in operative treatment of spondylolistheses: dorsal traction-reposition and anterior fusion (author's transl). Z Orthop Ihre Grenzgeb. 1982;120(3):343-347.

2. Xiao YX, Chen QX, Li FC. Unilateral transforaminal lumbar interbody fusion: a review of the technique, indications and graft materials. J Int Med Res. 2009;37(3):908-917.

3. Harris BM, Hilibrand AS, Savas PE et al. Transforaminal lumbar interbody fusion: The effect of various instrumentation techniques on the flexibility of the lumbar spine. Spine (Phila Pa 1976). 2004;29(4):E65-70.

4. Potter BK, Freedman BA, Verwiebe EG et al. Transforaminal lumbar interbody fusion: clinical and radiographic results and complications in 100 consecutive patients. J Spinal Disord Tech. 2005;18(4):337-346.

5. McAfee PC, Farey ID, Sutterlin CE et al. The effect of spinal implant rigidity on vertebral bone density. A canine model. Spine (Phila Pa 1976). 1991;16(6 Suppl):S190-197.

6. McAfee PC, Farey ID, Sutterlin CE et al. 1989 Volvo Award in basic science. Device-related osteoporosis with spinal instrumentation. Spine (Phila Pa 1976). 1989;14(9):919-926.

7. Goel VK, Lim TH, Gwon J et al. Effects of rigidity of an internal fixation device. A comprehensive biomechanical investigation. Spine (Phila Pa 1976). 1991;16(3 Suppl):S155-161.

8. Kabins MB, Weinstein JN, Spratt KF et al. Isolated L4- L5 fusions using the variable screw placement system: unilateral versus bilateral. J Spinal Disord. 1992;5(1):39-49.

9. Suk KS, Lee HM, Kim NH et al. Unilateral versus bilateral pedicle screw fixation in lumbar spinal fusion. Spine (Phila Pa 1976). 2000;25(14):1843-1847. 
10. Beringer WF, Mobasser JP. Unilateral pedicle screw instrumentation for minimally invasive transforaminal lumbar interbody fusion. Neurosurg Focus. 2006;20:E4.

11. Deutsch $\mathrm{H}$, Musacchio MJ Jr. Minimally invasive transforaminal lumbar interbody fusion with unilateral pedicle screw fixation. Neurosurg Focus. 2006;20(3):E10.

12. Xue H, Tu Y, Cai M. Comparison of unilateral versus bilateral instrumented transforaminal lumbar interbody fusion in degenerative lumbar diseases. Spine. 2012;12(3):209-215.

13. Foley KT, Holly LT, Schwender JD. Minimally invasive lumbar fusion. Eur Spine J. 2003;28(15 Suppl):S26-35.

14. Tuttle J, Shakir A, Choudhri HF. Paramedian approach for transforaminal lumbar interbody fusion with unilateral pedicle screw fixation. Technical note and preliminary report on 47 cases. Neurosurg Focus. 2006;20(3):E5.

15. Cao $Y$, Chen $Z$, Jiang $C$ et al. The combined use of unilateral pedicle screw and contralateral facet joint screw fixation in transforaminal lumbar interbody fusion. Eur Spine J. 2015;24(11):2607-2613.

16. Huang $P$, Wang $Y, X u J$ et al. Minimally invasive unilateral pedicle screws and a translaminar facet screw fixation and interbody fusion for treatment of single-segment lower lumbar vertebral disease: surgical technique and preliminary clinical results. J Orthop Surg Res. 2017;12(1):117.

17. Xiao SW, Jiang $H$, Yang LJ et al. Comparison of unilateral versus bilateral pedicle screw fixation with cage fusion in degenerative lumbar diseases: a meta-analysis. Eur Spine J. 2015;24(4):764-774.

18. Lin GX, Akbary K, Kotheeranurak V, Quillo-Olvera J , Jo HJ , Yang XW, Mahatthanatrakul A, Kim JS. Clinical and Radiologic Outcomes of Direct Versus Indirect Decompression With Lumbar Interbody Fusion: A MatchedPair Comparison Analysis World Neurosurg. 2018 Nov; 119:e898-e909. Disponible en: 10.1016/j.wneu.2018.08.003 [10 ago 2018] 